\title{
Particle therapy for non-small cell lung tumors: where do we stand? A systematic review of the literature
}

\author{
Krista C. J. Wink ${ }^{1 *}$, Erik Roelofs ${ }^{1}$, Timothy Solberg ${ }^{2}$, Liyong Lin ${ }^{2}$, Charles B. Simone $~ I I^{2}$, Annika Jakobi ${ }^{3}$, \\ Christian Richter ${ }^{3,4}$, Philippe Lambin ${ }^{1}$ and Esther G. C. Troost ${ }^{1}$ \\ ${ }^{1}$ Department of Radiation Oncology (MAASTRO Clinic), GROW - School for Oncology and Developmental Biology, Maastricht University Medical Centre, \\ Maastricht, Netherlands \\ ${ }^{2}$ Department of Radiation Oncology, Hospital of the University of Pennsylvania, Philadelphia, PA, USA \\ ${ }^{3}$ OncoRay - National Center for Radiation Research in Oncology, Faculty of Medicine and University Hospital Carl Gustav Carus, Technische Universität Dresden and \\ Helmholtz-Zentrum Dresden-Rossendorf, Dresden, Germany \\ ${ }^{4}$ German Cancer Consortium (DKTK) Dresden and German Cancer Research Center (DKFZ), Heidelberg, Germany
}

\section{Edited by:}

Zhongxing Liao, The University of Texas M. D. Anderson Cancer Center, USA

\section{Reviewed by:}

Deepak Khuntia, Western Radiation

Oncology, USA

Daniel Gomez, The University of Texas

M. D. Anderson Cancer Center, USA

${ }^{*}$ Correspondence:

Krista C. J. Wink, Department of

Radiation Oncology (MAASTRO

Clinic), Maastricht University Medical

Centre, Dr. Tanslaan 12, Maastricht

6229 ET, Netherlands

e-mail:krista.wink@maastro.n
This review article provides a systematic overview of the currently available evidence on the clinical effectiveness of particle therapy for the treatment of non-small cell lung cancer and summarizes findings of in silico comparative planning studies. Furthermore, technical issues and dosimetric uncertainties with respect to thoracic particle therapy are discussed.

Keywords: radiotherapy, NSCLC, photon, proton, C-ion, PSPT, PBS, IMPT

\section{INTRODUCTION}

Over the last few decades, improvements in surgical techniques and (chemo)radiotherapy have increased the survival rate in patients with lung cancer (1). Despite this, the survival of patients with locally advanced non-small cell lung cancer (NSCLC) remains poor, with a 5 -year overall survival (OS) of $15 \%$ for stage III NSCLC patients treated with concurrent chemoradiotherapy (2).

Prior studies have suggested that a higher tumor dose can result in increased locoregional control, and consequently in an improved disease-specific survival $(3,4)$. Different strategies to escalate the dose to the primary target volume have been studied, i.e., isotoxic dose enhancement, hyperfractionation, or dose-rescaling on an individual basis (5-7). However, the tumor-surrounding healthy tissues (healthy lung, heart, mediastinal structures, spinal cord, and brachial plexus) are often dose limiting.

Even though modern radiotherapy techniques (e.g., 3D conformal radiotherapy; 3D-CRT, or intensity-modulated radiation therapy; IMRT) decrease dose to surrounding tissues compared with 2D techniques, particle beam therapy may offer an even greater advantage. The favorable properties of charged particles in terms of dose distribution and radiobiological effectiveness make it an option worth investigating for the treatment of NSCLC. Treatment with protons or carbon ions can enable dose escalation beyond what can be achieved with the different photon techniques (8). There are, however, several difficulties with the delivery of particle beams to a tumor in the lung. These include the fact that the tumor and other anatomy may be moving, that variations exist in the water equivalent thickness of the beam path, and that the range uncertainties associated with pencil beam scanning (PBS) techniques are particularly sensitive to motion and setup errors $(9,10)$.

The aim of this article is to systematically review the currently available evidence on the clinical effectiveness and in silico comparative planning studies of particle therapy for the treatment of NSCLC. The technical issues and dosimetric uncertainties with respect to thoracic particle therapy are also discussed.

\section{METHODS}

The PubMed database was searched by two researchers to identify studies about particle therapy for NSCLC. Search items were lung, proton, particle, hadron, carbon, cancer, tumor, neoplasm, therapy, treatment, radiation therapy, radiotherapy, irradiation, and NSCLC. The search terms were used in different combinations and plural forms, and the search was limited to articles in English. References were screened for additional articles. Three types of studies were included: studies reporting on the outcomes of particle therapy for NSCLC, studies comparing dose distributions in (in silico) planning studies, and studies reporting on technical issues with particle therapy for NSCLC.

\section{RESULTS}

\section{CLINICAL OUTCOME}

All the studies reporting on clinical outcome of particle therapy for NSCLC were published by one of the following centers treating NSCLC patients with particle beams: the Loma Linda University 
Medical Center (LLUMC, Loma Linda, CA, USA), the MD Anderson Cancer Center (MDACC, Houston, TX, USA), the Proton Medical Research Center (PMRC, Tsukuba, Japan), the Hyogo Ion Beam Medical Center (HIBMC, Tatsuno, Japan), and the National Cancer Center Hospital East (NCCHE, Chiba, Japan). Due to overlapping study periods from the same institutions reporting on the same disease stages, it is reasonable to assume that the same patients were analyzed in multiple reports. This was, however, not always obvious because follow-up periods and patient numbers differed. Therefore, it was decided to include all the published studies in this review. In instances where the studied patient cohort was identical (same number of patients, patient characteristics, and follow-up period), only the most recent study reporting on clinical outcome was selected. Study characteristics are summarized in Table 1 (early-stage NSCLC) and Table 2 (advanced-stage NSCLC).

\section{Early-stage NSCLC}

Study descriptions. Fourteen studies investigated particle therapy for early-stage (stage I or II) NSCLC. The patients included were either medically inoperable or refused surgery, since surgery is still regarded as the first choice for treatment of Stage I NSCLC (11-13). Six studies reported the outcome of patients treated with protons only (14-19), four used carbon ions only (20-23), two studies reported outcome for both carbon ions and protons $(24,25)$, and two studies treated patients with a combination of photons and protons $(26,27)$. The most recent study is that of Kanemoto et al. (19). The researchers retrospectively evaluated disease control rates after high-dose proton beam therapy (PBT) in 74 patients with stage I NSCLC, with tumor doses of $66 \mathrm{~Gy}$ equivalent $(\mathrm{GyE})$ in $10-12$ fractions for peripheral tumors $(74 \%$ of all tumors) and $72.6 \mathrm{GyE}$ in 22 fractions for centrally located tumors. Another retrospective study from the same center (with different investigators), evaluated the outcome of PBT for 55 medically inoperable patients with stage I NSCLC, using the same total dose and fractionation (17). The study periods of both studies overlap, making it reasonable to assume that the patients included in the study by Nakayama et al. (17) were also assessed by the Kanemoto group (19).

In both studies by Iwata et al. (24, 25), early-stage NSCLC patients were treated with either carbon ions or protons. In their first report, the authors reported the outcome for 80 stage I NSCLC patients treated with $60-80 \mathrm{GyE}$ protons or $52.8 \mathrm{GyE}$ carbon ions during the period 2003-2007 (24). After restaging based on the AJCC seventh edition of the TNM classification and a longer inclusion and follow-up period (median 51 months), they analyzed the outcome for 70 cT2a/2bN0M0 NSCLC patients in a second study (25). Some patients in the cohort were, therefore, included in both the studies. The choice of particle type was based on beam availability (during part of the study, only proton beams were available), and if both particle types were available, a choice was made after comparison of dose-volume histograms for both modalities.

The American group of Bush et al. $(14,18,26)$ has performed three studies on proton beam irradiation for early-stage NSCLC in patients with contraindications to surgery or patients refusing surgery. In their first study, they treated 19 patients with insufficient pulmonary reserve or severe cardiac dysfunction with $51 \mathrm{GyE}$ of PBT to the gross tumor volume (GTV). In 18 patients with sufficient cardiopulmonary function, $45 \mathrm{~Gy}$ in $1.8 \mathrm{~Gy}$ fractions was delivered with photon irradiation, followed by a proton beam boost to the GTV of $28.8 \mathrm{GyE}$ in $1.8 \mathrm{GyE}$ fractions. In 2004 and 2013, updates of the first trial were published, focusing on stage I NSCLC patients treated with proton therapy only $(14,18)$. The study published in 2013 investigated the outcome of 111 earlystage NSCLC patients, with dose escalation from $51 \mathrm{GyE}$ in 10 fractions to $70 \mathrm{GyE}$ in 10 fractions during the course of the study (18). Nihei et al. (15) treated 37 stage I patients with PBT in Chiba, Japan. Ten of the included patients were enrolled in an earlier dose escalation study and all patients received 70-94 GyE in 20 fractions (fraction dose 3.5-4.7 GyE).

The use of carbon ions for early-stage NSCLC was investigated in four phase II studies by Japanese researchers in a similar study population (20-23). Sugane et al. (23) focused on patients of 80 years or older within the study cohorts of Miyamoto et al. $(21,22)$. The patients received $52.8 \mathrm{GyE}$ (stage IA) or $60 \mathrm{GyE}$ (stage IB) in four fractions during 1 week, or (if treated prior to 2000) $72 \mathrm{GyE}$ in 9 fractions over 3 weeks.

Overall survival and local control. The outcome of the selected studies for early-stage NSCLC is summarized in Table 3. The retrospective study by Nakayama et al. (17) observed 2-year OS and local control rates of 97.8 and $97.0 \%$, respectively. However, the study had a relatively short follow-up period (median 17.7 months). No difference in local recurrence between tumors located centrally or peripherally in the lung was observed. Kanemoto et al. (19) reported a 3-year OS of 76.7\% and a 3-year local control rate of $81.8 \%$ (stage IA $86.2 \%$, stage IB $67 \%$ ) with a median follow-up period of 31.0 months. The 3 -year local control rate was significantly better for peripherally located tumors: 88.4 versus $63.9 \%$ for centrally located tumors.

In their first study in 2010, Iwata et al. (24) reported the outcome for 80 patients treated with either protons or carbon ions with a median follow-up of 30.5 months. They observed 3-year OS and local control rates of 75 and $82 \%$, respectively. No significant difference in outcome was observed between the different treatment protocols. The authors also published the long-term outcome for cT2a and cT2b NSCLC patients (25). They reported a 4 -year local control rate of $75 \%$, and overall and progression-free survival rates of 58 and $46 \%$, respectively. No significant differences were observed based on T stage. The survival of the operable patients was better than for the medically inoperable patients.

Studies reporting on the use of carbon ions for early-stage lung cancer demonstrate 5-year OS and local control rates of 45 and $90 \%$, respectively (21), and of 30.7 and $95.8 \%$ (23) for patients aged 80 years or older.

Toxicity. The toxicity of PBT for early-stage NSCLC was found to be low, with the percentage of acute and late CTC grade $\geq 2$ adverse events generally $<10 \%$. This is not a surprising outcome, since stereotactic body radiation therapy (SBRT) with photons for stage I NSCLC also has a generally favorable toxicity profile (13). Mainly for peripheral tumors, few treatment related complications have been reported (28). The treatment of central tumors located close to vulnerable mediastinal structures, however, can 
Table 1 | Study descriptions early stage NSCLC.

\begin{tabular}{llcll}
\hline $\begin{array}{l}\text { Study (characteristics); } \\
\text { location }\end{array}$ & $\begin{array}{l}\text { Beam, energy, } \\
\text { technique }\end{array}$ & $\begin{array}{c}\text { Chemotherapy Fractionation schedule, } \\
\text { TTD } / n \text { or fr/OTT }\end{array}$ & $\begin{array}{l}\text { Patient selection } \\
\text { criteria/risk factors }\end{array}$
\end{tabular}

\section{PROTON BEAM ONLY}

(prospective, $n=37$ );

U.S. Loma Linda

Proton No

Photon + proton

PSc

Shioyama et al. (27)

(retrospective, $n=51$ );

Japan, Tsukuba

Proton, $250 \mathrm{MeV}, 6$ patients prior

PSc $(n=33)$

Photon + proton

$(n=18)$

No

$51 \mathrm{GyE} / 10 \mathrm{fr} / 2$ weeks PBT

Photons $45 \mathrm{~Gy} / 25 \mathrm{fr}$ + protons,

$28.8 \mathrm{GyE} / 16 \mathrm{fr} / 5$ weeks chemotherapy median fr dose $3.0 \mathrm{~Gy}$ (range (all advanced 2-6 Gy), median OTT 43 days stages)

Bush et al. (14)

Proton, PSc

No

(Phase II, $n=68$ ); U.S.

Loma Linda

Nihei et al. (15)

Proton, 150 or

No

(Retrospective, $n=37$ );

Japan, Chiba

$51 \mathrm{GyE} / 10 \mathrm{fr} / 2$ weeks $(n=22)$;

$60 \mathrm{GyE} / 10 \mathrm{fr} / 2$ weeks $(n=46)$
$70 \mathrm{GyE} / 20 \mathrm{fr} / 4-5$ weeks $(n=3)$; $80 \mathrm{GyE} / 20 \mathrm{fr} / 4-5$ weeks $(n=17)$; $88 \mathrm{GyE} / 20 \mathrm{fr} / 4-5$ weeks $(n=16)$; $94 \mathrm{GyE} / 20 \mathrm{fr} / 4-5$ weeks $(n=1)$

Hata et al. (16) (prospective, $n=21$ ), Japan, Tsukuba

Nakayama et al. (17) Proton, No (retrospective, $n=55$ ) (58 tumors) Japan, 155-250 MeV, PSC

Tsukuba

Bush et al. (18) Proton, PSc No

(Phase 2, $n=111$ ); U.S

Loma Linda

Kanemoto et al. (19)

Proton,

No

(Retrospective, $n=74$,

80 tumors), Japan,

$155-250 \mathrm{MeV}$ PSC

Tsukuba

\section{C-ION (COMBINED WITH PROTON BEAM)}

Iwata et al. (24)

(retrospective, $n=80$ ),

Japan, Hyogo

Proton $150 \mathrm{MeV}, \quad \mathrm{No}$

PSC

C-ion $320 \mathrm{MeV}$,

PSC

Proton $150 \mathrm{MeV}$, No

Iwata et al. (25)

\{retrospective [partially

subgroup of cohort

Iwata (24)], $n=70$ \},

Japan, Hyogo
PSc

C-ion $320 \mathrm{MeV}$,

PSC
Peripheral tumors $66 \mathrm{GyE} / 10$ $\mathrm{fr} / 2$ weeks $(n=41)$, central tumors $72.6 \mathrm{GyE} / 22 \mathrm{fr} / 4.5$ weeks $(n=17)$

Dose escalation during study, $51 \mathrm{GyE} / 10 \mathrm{fr} / 2$ weeks $(n=29)$, $60 \mathrm{GyE} / 10 \mathrm{fr} / 2$ weeks $(n=56)$, $70 \mathrm{GyE} / 10 \mathrm{fr} / 2$ weeks $(n=26)$

Peripheral tumors 66 GyE/10-12 fr/2-2.5 weeks $(n=59)$, central tumors $72.6 \mathrm{GyE} / 22 \mathrm{fr} / 4.5$ weeks $(n=21)$

Proton: $80 \mathrm{GyE} / 20 \mathrm{fr} / 4$ weeks

$(n=20), 60 \mathrm{GyE} / 10 \mathrm{fr} / 2$ weeks

$(n=37)$,

C-ion: $52.8 \mathrm{GyE} / 4 \mathrm{fr} / 1$ week $(n=23)$

Proton: $80 \mathrm{GyE} / 20 \mathrm{fr} / 4$ weeks ( $n=14), 60 \mathrm{GyE} / 10 \mathrm{fr} / 2$ weeks ( $n=20), 66 \mathrm{GyE} / 10 \mathrm{fr} / 2$ weeks ( $n=8), 70.2 \mathrm{GyE} / 26 \mathrm{fr} / 5.5$ weeks $(n=1)$
NSCLC stage I-IIIA; medically

inoperable/refused surgery

Stage I, 27; Stage II,

2; Stage IIIA, 8

NSCLC, medically

inoperable/refused surgery

NSCLC stage I, medically inoperable $(n=63) /$ refused surgery $(n=5)$

NSCLC stage I; medically inoperable $(n=23) /$ refused surgery $(n=14)$, tumor size $\leq 5 \mathrm{~cm}, \mathrm{pO}_{2} \geq 60$ torr, Zubrad PS 0-2

NSCLC stage I, medically inoperable $(n=9) /$ refused surgery ( $n=12)$, ECOG PS $0-2$, no previous RT or chemotherapy for NSCLC

NSCLC stage I; EORTC PS $0-2$, medically inoperable $(n=52)$ /refused surgery $(n=3)$, Exclusion: pleural effusion, tumor close to stomach/esophagus

NSCLC stage I, medically inoperable/refused surgery

Stage IA, 47; Stage IB, 64

NSCLC stage I

Stage IA, 59;

Stage IB, 21

NSCLC stage I, medically

inoperable $(n=47) /$ refused

Stage IA, 42

surgery $(n=43)$, WHO PS 0-2,

no history lung cancer or previous chest RT/chemo

NSCLC cT2a/T2bNOMO,

medically inoperable

Stage IB, 47:

( $n=40)$ /refused surgery

$(n=30)$, WHO PS 0-2, no

history lung cancer or previous

chest RT/chemotherapy 
Table 1 | Continued

\begin{tabular}{|c|c|c|c|c|c|}
\hline $\begin{array}{l}\text { Study (characteristics); } \\
\text { location }\end{array}$ & $\begin{array}{l}\text { Beam, energy, } \\
\text { technique }\end{array}$ & Chemotherapy & $\begin{array}{l}\text { Fractionation schedule, } \\
\text { TTD/n or fr/OTT }\end{array}$ & $\begin{array}{l}\text { Patient selection } \\
\text { criteria/risk factors }\end{array}$ & Stage distribution \\
\hline & & & $\begin{array}{l}\text { C-ion: } 52.8 \mathrm{GyE} / 4 \mathrm{fr} / 1 \text { week } \\
(n=16), 66 \mathrm{GyE} / 10 \mathrm{fr} / 2 \text { weeks } \\
(n=8), 68.4 \mathrm{GyE} / 9 \mathrm{fr} / 2 \text { weeks } \\
(n=3)\end{array}$ & & \\
\hline $\begin{array}{l}\text { Miyamoto et al. (21) } \\
\text { (phase II, } n=79 \text { ), } \\
\text { Japan, Chiba }\end{array}$ & $\begin{array}{l}\text { C-ion, 290-350- } \\
400 \mathrm{MeV} \\
\text { PSc }\end{array}$ & No & $\begin{array}{l}52.8 \mathrm{GyE} / 4 \mathrm{fr} / 1 \text { week (IA) } \\
60 \mathrm{GyE} / 4 \mathrm{fr} / 1 \text { week (IB) }\end{array}$ & $\begin{array}{l}\text { NSCLC stage I, medically } \\
\text { inoperable/refused surgery, } \\
\text { WHO PS 0-2, no history of RT } \\
\text { to target, no prior } \\
\text { chemotherapy }<4 \text { weeks }\end{array}$ & $\begin{array}{l}\text { Stage IA, 42; } \\
\text { Stage IB, } 37\end{array}$ \\
\hline $\begin{array}{l}\text { Sugane et al. (23) } \\
\text { (phase II, } n=28,29 \\
\text { tumors), Japan, Chiba }\end{array}$ & $\begin{array}{l}\text { C-ion, 290-350- } \\
400 \mathrm{MeV} \\
\text { PSc }\end{array}$ & No & $\begin{array}{l}\text { 72.0 GyE/9 fr/3 weeks (all, } \\
\text { 1999-2000, } n=12), 60.0 \mathrm{GyE} / 4 \\
\mathrm{fr} / 1 \text { week (IA, } n=11), 52.8 \mathrm{GyE} / 4 \\
\mathrm{fr} / 1 \text { week }(\mathrm{IB}, n=6)\end{array}$ & $\begin{array}{l}\text { NSCLC stage I; elderly, aged } \\
\geq 80 \text { years }\end{array}$ & $\begin{array}{l}\text { Stage IA, 12; } \\
\text { Stage IB, } 17\end{array}$ \\
\hline
\end{tabular}

ECOG, Eastern Cooperative Oncology Group; EORTC, European Organization for Research and Treatment of Cancer; OTT, overall treatment time; PS, performance score; PSC, passive scattering; RT, radiotherapy; TTD, total tumor dose; WHO, World Health Organization.

cause a higher rate of severe toxicity (29). The LungTech study (NCT01795521) is currently recruiting patients for a phase II trial of hypofractionated photon radiotherapy $(60 \mathrm{~Gy}$ in 8 fractions given on alternating days) for centrally located tumors in patients with inoperable disease. Using PBT, Bush et al. (18) reported a low incidence of late rib fractures (4\%), and in all of the cases, the tumor was located close to the chest wall. In contrast, other studies reported a relatively high incidence of Grade $\geq 2$ rib fractures of $14-27 \%(19,24,25)$. In the experience of Iwata et al., the rib fractures mainly occurred in patients treated with only one beam portal $(24,25)$. These patients received a rib dose of $70-100 \%$ of the isocenter dose.

\section{Locally advanced NSCLC}

Study descriptions. Three studies have reported on the outcome of proton therapy for locally advanced NSCLC. The retrospective study of Oshiro et al. (30) evaluated the outcome of patients with stage III NSCLC after treatment with protons, without concurrent chemotherapy. The cohort consisted of 57 patients treated with a median dose of $74 \mathrm{GyE}$ (range 50-85 GyE) in 2 GyE fractions (range 2-6.6 GyE). A respiratory gating system was used, and patients were immobilized with a body cast. Due to patient comorbidities, old age or patient refusal, concurrent chemotherapy was not administered. Fourteen patients received induction chemotherapy, however, without a tumor response. Nakayama et al. (31) reported on 35 stage II and III NSCLC patients treated in Tsukuba between 2001 and 2008 with protons to a median dose of $78.3 \mathrm{GyE}$. All patients were unsuitable for $(n=31)$ or refused chemotherapy or surgery $(n=4)$. Chang et al. (32) performed a phase II study at MD Anderson Cancer Center on the use of protons with concurrent chemotherapy for unresectable Stage III non-small-cell lung cancer. Forty-four stage III NSCLC patients were included and treated with $74 \mathrm{GyE}$ using passively scattered protons, combined with weekly carboplatin and paclitaxel. Treatment simulation with four-dimensional CT was undertaken and an internal gross tumor volume (iGTV) was defined to account for tumor motion. Verification 4D CT scans were obtained in the third or fourth week of treatment. In case of significant changes regarding patient anatomy or tumor shrinkage, a new treatment plan was constructed [see Ref. (33)].

Overall survival and local control. After a median follow-up of 22.2 months (for surviving patients), Oshiro et al. (30) reported 1- and 2-year OS rates of 65.5 and 39.4\%, respectively (Table 4). Progression-free survival and local control rates were $24.9 \%$ and $64.1 \%$ at 2 years, respectively. Distant metastases were most often the initial sites of recurrence. Induction chemotherapy did not have a significant effect on the OS.

For the NSCLC patients included in the study by Nakayama et al. (31), the 2-year OS was 58.9\% and the local progression-free survival at 2 years was $65.9 \%$. Four patients (11.4\%) developed a recurrence within the irradiated primary tumor volume, 10 
Table 2 | Study descriptions advanced-stage NSCLC.

\begin{tabular}{|c|c|c|c|c|c|}
\hline $\begin{array}{l}\text { Study (characteristics); } \\
\text { location }\end{array}$ & $\begin{array}{l}\text { Beam, energy, } \\
\text { technique }\end{array}$ & Chemotherapy & $\begin{array}{l}\text { Fractionation schedule, } \\
\text { TTD/n or fr/OTT }\end{array}$ & $\begin{array}{l}\text { Patient selection } \\
\text { criteria/risk factors }\end{array}$ & Stage distribution \\
\hline \multicolumn{6}{|l|}{ PROTON BEAM ONLY } \\
\hline $\begin{array}{l}\text { Bush et al. (26) (prospective, } \\
n=37 \text { ); U.S. Loma Linda }\end{array}$ & $\begin{array}{l}\text { Proton } \\
\text { Photon + proton PSc }\end{array}$ & No & $\begin{array}{l}51 \mathrm{GyE} / 10 \mathrm{fr} / 2 \text { weeks } \\
\text { Photons, } 45 \mathrm{~Gy} / 25 \mathrm{fr}+\text { protons, } \\
28.8 \mathrm{GyE} / 16 \mathrm{fr} / 5 \text { weeks }\end{array}$ & $\begin{array}{l}\text { NSCLC stage I-IIIA; medically } \\
\text { inoperable/refused surgery }\end{array}$ & $\begin{array}{l}\text { Stage I, 27; Stage II, 2; } \\
\text { Stage IIIA, } 8\end{array}$ \\
\hline $\begin{array}{l}\text { Shioyama et al. (27) } \\
\text { (retrospective, } n=51 \text { ); Japan, } \\
\text { Tsukuba }\end{array}$ & $\begin{array}{l}\text { Proton, } 250 \mathrm{MeV}, \\
\text { PSc }(n=33) \\
\text { Photon + proton } \\
(n=18)\end{array}$ & $\begin{array}{l}6 \text { patients prior chemotherapy } \\
\text { (all advanced stages) }\end{array}$ & $\begin{array}{l}\text { Median TD } 76 \text { Gy (range 49-93 Gy), } \\
\text { median fr dose 3.0 Gy (range } \\
\text { 2-6 Gy), median OTT } 43 \text { days }\end{array}$ & $\begin{array}{l}\text { NSCLC, medically inoperable/refused } \\
\text { surgery }\end{array}$ & $\begin{array}{l}\text { Stage IA/IB, 9/19; Stage } \\
\| A / I B, 3 / 6 ; \text { Stage } \\
\text { IIA/IIB, 8; Stage IV, 1, } \\
\text { recurrent disease, } 5 \text {. }\end{array}$ \\
\hline $\begin{array}{l}\text { Chang et al. (32) (phase II, } \\
n=44 \text { ), U.S., Houston }\end{array}$ & Proton, PSc & $\begin{array}{l}\text { Concurrent, weekly carboplatin } \\
(2 \mathrm{AUC})+\text { paclitaxel }\left(50 \mathrm{mg} / \mathrm{m}^{2}\right) \\
(n=44) \text {. (neo) Adjuvant } \\
\text { chemotherapy allowed }(n=19)\end{array}$ & $74 \mathrm{GyE} / 37 \mathrm{fr} / 7.5$ weeks & $\begin{array}{l}\text { NSCLC Stage III, } \\
\text { Unresectable/medically inoperable, } \\
\text { KPS } 70-100 \text {, weight loss not }>10 \% \\
\text { during }<6 \text { months before diagnosis }\end{array}$ & $\begin{array}{l}\text { Stage IIIA, 21; Stage } \\
\text { IIIB, } 23\end{array}$ \\
\hline $\begin{array}{l}\text { Nakayama et al. (31) } \\
\text { (retrospective, } n=35 \text { ), Japan, } \\
\text { Tsukuba }\end{array}$ & $\begin{array}{l}\text { Proton, } \\
155-250 \mathrm{MeV}, \mathrm{PSc}\end{array}$ & No & $\begin{array}{l}77 \mathrm{GyE} / 35 \mathrm{fr} / 7 \text { weeks }(n=13), \\
83.6 \mathrm{GyE} / 38 \mathrm{fr} / 7.5 \text { weeks }(n=7) \text {, } \\
72.6 \mathrm{GyE} / 22 \mathrm{fr} / 4.5 \text { weeks }(n=6) \text {, } \\
74 \mathrm{GyE} / 37 \mathrm{fr} / 7.5 \text { weeks }(n=3) \\
\text { other }(n=6)\end{array}$ & $\begin{array}{l}\text { NSCLC Stage II/III, medically } \\
\text { inoperable/refused surgery, EORTC } \\
\text { PS 0-2, unsuitable for/refusal } \\
\text { chemotherapy. Exclusion: pleural } \\
\text { effusion, tumor close to } \\
\text { stomach/esophagus }\end{array}$ & $\begin{array}{l}\text { Stage IIA, 2; IIB, 3; } \\
\text { Stage IIIA, 12, Stage } \\
\text { IIIB, } 18\end{array}$ \\
\hline $\begin{array}{l}\text { Xiang et al. (34) (prospective, } \\
n=84 \text { ), U.S. Houston }\end{array}$ & Proton, PSc & $\begin{array}{l}\text { Concurrent, weekly carboplatin } \\
(2 \mathrm{AUC})+\text { paclitaxel }\left(50 \mathrm{mg} / \mathrm{m}^{2)}\right. \\
(n=84) \text {. (neo) Adjuvant } \\
\text { chemotherapy allowed }(n=22)\end{array}$ & $74 \mathrm{GyE} / 37 / 7.5$ weeks & $\begin{array}{l}\text { Stage III NSCLC, unresectable, } \\
\text { availability of pre- and post-treatment } \\
\text { PET-CT images }\end{array}$ & NR \\
\hline $\begin{array}{l}\text { Oshiro et al. (30) } \\
\text { (retrospective, } n=57) \text {, Japan, } \\
\text { Tsukuba }\end{array}$ & $\begin{array}{l}\text { Proton, } 200 \mathrm{MeV} \text {, } \\
\text { PSc }\end{array}$ & $\begin{array}{l}\text { No concurrent chemotherapy, } \\
\text { induction chemotherapy: } n=14\end{array}$ & $\begin{array}{l}\text { Median } 74 \text { GyE (50-84.5 GyE), } \\
\text { median fr dose 2.0 GyE } \\
(2.0-6.6 \mathrm{GyE})\end{array}$ & Stage III NSCLC & $\begin{array}{l}\text { Stage IIIA, 24; } \\
\text { Stage IIIB, } 33\end{array}$ \\
\hline
\end{tabular}

AUC, area under the curve; EORTC, European Organization for Research and Treatment of Cancer; KPS, Karnofsky Performance Status; NR, not reported; PS, performance score; PSC, passive scattering; TTD, total tumor dose. 


Study (characteristics)i

\section{location}

\section{PROTON BEAM ONLY}

\section{Bush et al. (26) (prospective}

$n=35)$ : U.S. Loma Linda

Shioyama et al. (27) (retrospective, $n=51)$; Japan, Tsukuba

\section{Median 14 month}

(range 3-45)

Median 30 months (range 18-153)

Bush et al. (14) (Phase II, $n=68$ ); $\quad$ At least 12 months U.S. Loma Linda

Nihei et al. (15) (Retrospective, $n=37) ;$ Japan, Chiba

Median 24 months (range 3-62) Japan, Tsukuba (range 10-54)

Nakayama et al. (17) (retrospective, Median 17.7 months $n=55$ ) (58 tumors) Japan, Tsukuba (range 1.4-53.3)

Bush et al. (18) (phase 2, $n=111$ ); $\quad$ Median 48 months U.S. Loma Linda

Kanemoto et al. (19) (retrospective, Median 31 months $n=74,80$ tumors), Japan, Tsukuba (range 7.3-104.3)
Hata et al. (16) (prospective, $n=21$ ), Median 25 months
2 years $31 \%$ (Stage I: $39 \%$ )

\section{2 years $87 \%$}

2 years $62 \%, 5$ years $29 \%$

2 years Stage I/II: $55 \%$,

Stage IA 88\%, IB 47\%

5 years Stage $1 / / 123 \%$

IA $70 \%$, IB $16 \%$

3 years $44 \%: 51$ GyE: $27 \%$ 60 GyE: $55 \%$

2 years $84 \%$

years $74 \%$ (Stage IA 100\%， 2 years 95\% (Stage IA 19\% ${ }^{\mathrm{a}}$ IB $47 \%$ ) $100 \%$, IB 90\%)

2 years $97.8 \%$

4 years: $51 \mathrm{GyE} 18 \%, 60 \mathrm{GyE}$ $32 \%, 70$ Gy $51 \%$ 4 years OS peripheral T1: $60 \%$

3 years $76.7 \%, 5$ years $65.8 \%$
5 years $57 \%$ (Stage IA $16 \%^{\text {a }}$ $89 \%$, IB 39\%

3 years $74 \%$ (Stage IA: 3 years $31 \% \quad 3$ years $72 \%$ $87 \%$, IB: $49 \%$ )

1 years $91 \%, 2$ years $19 \%^{\circ}$ $80 \%$

\section{2 years 63\% (Stage G2 RP: $5.7 \%$}

I: $86 \%$

5 years 37\% (Stage Acute: lung tox $\leq \mathrm{G} 1: 92 \%, \mathrm{G} 2: 6 \%$, IA 89\%, IB 17\%) G3: $2 \%$

\section{C-ION (COMBINED WITH PROTON BEAM)}

$n=80)$, Japan, Hyogo
Iwata et al. (24) (retrospective,

\section{Median 30.5 months}

(range 18-66)

\section{3 years $75 \%$ (Stage IA 74\%,}

IB 76\%)

3 years OS by dose: $80 \mathrm{GyE}$ $90 \%, 60 \mathrm{GyE} 61 \%, 52.8 \mathrm{GyE}$ $86 \%$
2 years $97 \%$

$0 \%$

4 years: 60 GyE $45 \%, \quad 4$ years 70 Gy $74 \%$ $81 \%$

3 years $81.8 \%, 5$ years NR $81.8 \%$ $86.2 \%$, IB $67 \%$ peripheral T1

3 years Stage IA

\section{1 year $73 \%, 2$ years} $58 \%$

\section{NR}

Acute: G1: $84 \%$ (mostly dermatitis) No $\geq$ grade 2

Late: lung toxicity (pneumonitis/pleural effusion): G2 $8 \%$ or G3: $8 \%$

2 years 79\% (Stage Acute: hematological G1-2: 14\%. |A 89\%, IB 70\%) Dermatitis G1: 19\%, RP G2: 5\% Late: G2: $10 \%$ (subcutaneous induration/myositis)

2 years $88.7 \%, \quad$ Acute: lung (pneumonitis) G1: $25.4 \%$, 3 years $78.9 \%$ G2: $3.6 \%, \mathrm{G} 3: 3.6 \%$ Late: rib fracture: $1.8 \%$

NR Rib fractures: $3.6 \%$, no other $\geq \mathrm{G} 2$ adverse events

3 years 58.6\%, Acute: G2 skin: $2.5 \%$, G2 esophagitis: 5 years $52.5 \%$

$1.3 \%$, G3 pneumonitis: $1.3 \%$ Late: G3 RP: $1.3 \%$, G3 skin ulcer $1.3 \%, \mathrm{G} 4$ rib fracture $13.8 \%$

\section{3 years 54\% (Stage Lung toxicity: G2 RP: 11\%, G3 RP} $87 \%$, IB $77 \%$ )
IA $67 \%$, IB $46 \%$ ) $13 \%$

3 years LC by dose:

$80 \mathrm{GyE} 83 \%, 60 \mathrm{GyE}$

Skin: dermatitis G2/3: 16\%

G2 rib fracture: $23 \%$ 
Table 3 | Continued

\section{Study (characteristics)}

FU

os

LC

DM

PFS

Toxicity

Iwata et al. (25) \{retrospective

[partially subgroup of cohort Iwata

Median 51 months

4 years 58\% (T2a 53\%, T2b

4 years $75 \%$ (T2a

$20 \%^{\mathrm{a}}$

4 years $46 \%$ (T2a

radiation pneumonitis 3\%

(24)], $n=70\}$, Japan, Hyogo

Operable patients $(n=30): \quad$ Operable patients

4 years $72 \%$

$(n=30): 4$ years $73 \%$

Miyamoto et al. (20) (phase I/II,

$n=81,82$ tumors), Japan, Chiba

Median 52.6 months

5 years $42 \%$ (Stage IA 64.4\%, 23\% local recurrence Stage IB 22\%)

at 6.2-27.2 months

after start RT

Miyamoto et al. (21) (phase II, $n=79)$, Japan, Chiba

Median 38.6 months

5 years $45 \%$ (Stage IA: 62\%, 5 years 90\% (Stage IA $27 \%$ a

$43 \%$, Т2b $52 \%$

G2/3/4 dermatitis $7 \%$, Rib fracture G2

Operable patients

$27 \%$, soft tissue fibrosis G2 6\%

(range 2.5-72.2)

IB 25\%)

$98 \%$, IB $80 \%$ )

$(n=30): 4$ years $46 \%$

Acute: lung G2: $6.2 \%$, G3: $3.7 \%$ Late: lung G2: $1.2 \%$

Acute: lung G2: $1.3 \%$

Late: lung G2: $1.3 \%$

No G3 toxicity

Miyamoto et al. (22) (phase II,

$n=50,51$ tumors), Japan, Chiba

Median 59.2 months

5 years $50 \%$ (Stage IA 55.2\%, 5 years $95 \%$

$28 \%^{a}$

NR

(range 6-83)

IB 42.9\%)

NR

2 years $64.3 \%, 5$ years $30.7 \% \quad 2$ and 5 years: $95.8 \% \quad N R$

29 tumors), Japan, Chiba

(Stage IB 21.2\%)

(Stage IA 1

91.7\%). Tumor $>4 \mathrm{~cm}$ :

5 years LC $80 \%$

Acute: lung toxicity: G1: $2 \%, \mathrm{G} 2: 2 \%$. Skin G1: 100\%

Late: lung G1: 96\%, G2: 4\%. Skin

G1: $98 \%$

Acute: no lung toxicity. Skin G1: $96 \%$

Late: lung toxicity G1: $96 \%$. Skin G1: $100 \%$

${ }^{a}$ Own calculation, time-point unknown.

DM, distant metastases; FU, follow-up; G, CTC Grade; LC, local control; NR, not reported; OS, overall survival; PFS, progression free survival; RP, radiation pneumonitis; RT, radiotherapy. 
Table 4 | Study outcomes advanced-stage NSCLC

\begin{tabular}{|c|c|c|c|c|c|c|}
\hline Study (characteristics); location & FU & os & LC & DM & PFS & Toxicity \\
\hline \multicolumn{7}{|l|}{ PROTON BEAM ONLY } \\
\hline $\begin{array}{l}\text { Bush et al. (26) (prospective, } \\
n=35 \text { ); U.S. Loma Linda }\end{array}$ & $\begin{array}{l}\text { Median } 14 \text { months } \\
\text { (range 3-45) }\end{array}$ & 2 years $31 \%$ (IIIA 13\%) & 2 years $87 \%$ & $14 \%^{a}$ & $\begin{array}{l}2 \text { years 63\% (IIIA } \\
19 \%)\end{array}$ & G2 RP: $5.7 \%$ \\
\hline $\begin{array}{l}\text { Shioyama et al. (27) (retrospective, } \\
n=51 \text { ); Japan, Tsukuba }\end{array}$ & $\begin{array}{l}\text { Median } 30 \text { months } \\
\text { (range 18-153) }\end{array}$ & $\begin{array}{l}2 \text { years } 62 \%, 5 \text { years } 29 \% \\
2 \text { years Stage III/IV } 62 \%, \\
5 \text { years Stage III/IV } 0 \%\end{array}$ & $\begin{array}{l}5 \text { years } 57 \% \text { (total } \\
\text { group) }\end{array}$ & $16 \%^{a}$ & $\begin{array}{l}5 \text { years } 37 \% \text { (total } \\
\text { group) }\end{array}$ & $\begin{array}{l}\text { Acute: lung tox } \leq \mathrm{G} 1: 92 \%, \mathrm{G} 2: 6 \% \text {, } \\
\text { G3: } 2 \%\end{array}$ \\
\hline $\begin{array}{l}\text { Chang et al. (32) (phase II, } n=44 \text { ), } \\
\text { U.S., Houston }\end{array}$ & $\begin{array}{l}\text { Median 19.7mnd } \\
\text { (range 6.1-44.4) }\end{array}$ & 1 year $86 \%$ & $\begin{array}{l}\text { Local recurrence rate } \\
20.5 \%\end{array}$ & $43 \%$ & 1 year $63 \%$ & $\begin{array}{l}\text { Acute: } \mathrm{CT} \text { related G4 tox: } 11.4 \% \text {. G3 } \\
\text { dermatitis: } 11.4 \%, \mathrm{G} 3 \text { esophagitis: } \\
11.4 \% \text {, G3 RP: } 2.3 \% \text {. No G4 toxicity } \\
\text { Late: pulmonary/pleural fistula: } 2.3 \%\end{array}$ \\
\hline $\begin{array}{l}\text { Nakayama et al. (31) (retrospective, } \\
n=35 \text { ), Japan, Tsukuba }\end{array}$ & NR & 1 year $81.8 \%, 2$ years $58.9 \%$ & $\begin{array}{l}1 \text { year } 93.3 \%, 2 \text { years } \\
65.9 \%\end{array}$ & $20 \%$ & $\begin{array}{l}1 \text { year } 59.6 \% \\
2 \text { years } 29.2 \%\end{array}$ & $\begin{array}{l}\text { Lung: G1: } 25.7 \%, G 2: 14.3 \% \text {. G2 } \\
\text { esophagitis: } 2.9 \% \text {. No } \geq \text { G3 toxicity }\end{array}$ \\
\hline $\begin{array}{l}\text { Xiang et al. (34) (prospective, } \\
n=84 \text { ), U.S. Houston }\end{array}$ & $\begin{array}{l}\text { Median } 19.2 \text { months } \\
(6.1-52.4)\end{array}$ & 3 years $37.2 \%$ & 2 years $83 \%$ & $\begin{array}{l}39 \% 3 \text { years } \\
\text { DMFS } 35.4 \%\end{array}$ & 3 years $31.2 \%$ & NR \\
\hline $\begin{array}{l}\text { Oshiro et al. (30) (retrospective, } \\
n=57 \text { ), Japan, Tsukuba }\end{array}$ & Median 16.2 months & 1 year $65.5 \%, 2$ years $39.4 \%$ & $\begin{array}{l}1 \text { year } 79.1 \%, 2 \text { years } \\
64.1 \%\end{array}$ & $47 \%^{a}$ & $\begin{array}{l}1 \text { year } 36.2 \% \\
2 \text { years } 24.9 \%\end{array}$ & $\begin{array}{l}\text { Acute: lung (RP) G2: 7\%, G3: } 1.8 \% \text {, } \\
\text { G4: } 1.8 \%, \text { G5: } 1.8 \% \text {. Esophagitis G2: } \\
\text { 1.8\% } \\
\text { Late: RP G2: } 6.3 \% \text {. Hemoptysis G5: } \\
2.1 \%\end{array}$ \\
\hline
\end{tabular}

a Own calculation, time-point unknown.

DM, distant metastases; DMFS, distant metastasis free survival; FU, follow-up; G, CTC Grade; LC, local control; NR, not reported; OS, overall survival; PFS, progression free survival; RP, radiation pneumonitis; $R T$, radiotherapy. 
patients $(35 \%)$ developed a regional recurrence, and 7 patients (20\%) developed distant metastases. Chang et al. (32) added concurrent chemotherapy to PBT and reported overall and progression-free survival of 86 and 63\%, respectively, at 1 year, and a median OS time of 29.4 months. Again, distant metastases were the most frequent site of failure (19 patients, 43\%). Only four patients $(9.1 \%)$ had an isolated local recurrence. OS and progression-free survival (PFS) were better than those reported by Oshiro et al. (30), possibly due to the added effects of the concurrent chemotherapy or the pretreatment condition of the patients. While the patients included by Chang et al. (32) were fit enough to receive (concurrent) chemotherapy, most of those described by Oshiro et al. (30) were not (mostly due to old age and comorbidities). In an updated analysis of an expanded cohort of 84 patients treated at MD Anderson Cancer Center with concurrent chemotherapy and PBT for locally advanced NSCLC that assessed the correlation between post-treatment FDG uptake on PET/CT scan and clinical outcomes, including survival, Xiang et al. (34) reported a similar median survival time of 29.9 months. At 3 years, the local recurrence-free survival (LRFS) rate was $34.8 \%$, distant metastasis-free survival (DMFS) rate was $35.4 \%$, PFS rate was $31.2 \%$, and the OS rate was $37.2 \%$. Furthermore, post-treatment SUV was found on multivariate analysis to be independently prognostic for LRFS, DMFS, PFS, and OS (all $p<0.05)$.

Toxicity. Acute treatment toxicity was generally mild. Oshiro et al. (30) reported Grade $\geq 3$ lung toxicity in three patients (5\%): one patient had Grade 3 pneumonitis effectively treated with steroids, one patient had to discontinue treatment due to Grade 4 pneumonitis, and one patient died of pneumonitis during treatment (Grade 5). Both patients with the severe pneumonitis had severe preexisting interstitial pneumonitis prior to the diagnosis of NSCLC and, therefore, were deemed unsuitable for surgery or conventional (photon) radiotherapy. Three patients developed Grade $\geq 3$ late toxicity: two patients with Grade 3 dyspnea, and one patient with Grade 5 hemoptysis (after repeated biopsy of the irradiated bronchus).

In the study by Chang et al. (32), toxicity was primarily related to the administered chemotherapy and consisted mainly of Grade 2 and 3 bone marrow suppression. A further five patients (11.4\%) experienced Grade 3 esophagitis, one patient (2.3\%) developed Grade 3 pneumonitis, and five patients (11.4\%) had Grade 3 dermatitis. One patient developed a pulmonary/pleural fistula. No Grade 5 toxicity occurred. Toxicity was not reported in the expanded analysis by Xiang et al. Nakayama et al. (31) reported no Grade $\geq 3$ toxicities.

\section{PROTON THERAPY TECHNIOUES AND CONSIDERATIONS FOR TREATMENT PLANNING AND DELIVERY}

Protons can be delivered with two different radiation techniques: passive scattering proton therapy (PSPT) or PBS. In PSPT, the tumor volume is irradiated as a whole, using collimators and compensators for dose conformality. With PBS, the target volume is scanned spot-by-spot with a narrow proton beam, enabling intensity-modulated proton therapy (IMPT). Most institutions treating NSCLC with protons use the passive technique as it is the most widely available and moreover less sensitive to breathing motion than PBS.

Matney et al. (35) quantified and compared the effects of respiratory motion on clinically delivered IMRT and re-calculated PSPT plans for 20 stage II-IIIb NSCLC patients. For a respiratory motion of up to $17 \mathrm{~mm}$, target coverage was maintained for both IMRT and PSPT. Only two of the studied comparative parameters, lung V5 and spinal cord Dmax, were statistically significantly better when using PSPT. The authors concluded that PSPT may not be more susceptible to respiratory motion than IMRT.

Passive scattering proton therapy also leads to a non-conformal dose distribution at the proximal edge of the field. Conformality is better for the PBS technique, but the interplay between the intrafractional tumor motion and the scanned proton beams can have detrimental effects on the dose distribution. This so-called "interplay effect" can result in severe under- or overdosage: a spot can be irradiated several times or not at all. Thus, the dose is not only inhomogeneous at the edges but also inside the target, and therefore, cannot be accounted for by simply adding a surrounding margin (36). This is crucial especially for full IMPT that, in contrast to the single field uniform dose (SFUD) technique, results in completely inhomogeneous dose distributions per field. To our knowledge, only the MD Anderson Cancer Center has started to treat NSCLC with PBS using the SFUD technique, limiting the treatment to a subset of tumors with motion amplitudes below $5 \mathrm{~mm}$. Li et al. (37) investigated the extent of the interplay effect on IMPT delivery and concluded that for the specific system at MDACC, the this effect may not be a primary concern. Grassberger et al. (38) and Dowdell et al. (39) have reported that the magnitude of the interplay effect depends on patient parameters such as motion amplitude and beam delivery parameters such as spot size. Several techniques (rescanning, gating, tracking) trying to compensate for the interplay effect are topic of intensive research in several groups worldwide. For IMPT, the robustness and a geometrical tumor tracking method mitigating to motion were evaluated in 7 NSCLC patients treated to $45 \mathrm{GyE}$ in 3 fractions (40). Patients had in total nine peripheral stage I lung tumors that were imaged with $4 \mathrm{D}-\mathrm{CT}$, and the combined dose distribution was summed using deformable image registration. Additionally, plans were constructed in which the proton beams were geometrically shifted without adjusting the beam energy. In 6 tumors showing a displacement of $<1 \mathrm{~cm}, 97-100 \%$ of the GTV was covered by $95 \%$ of the prescribed dose. For the remaining three patients, this was 95, 82, and 51\%, respectively; subsequently, the application of a geometrical tracking method improved this to 100 , 98 , and $97 \%$, respectively. The authors concluded that the simple tracking method was valuable for improving GTV coverage. The practicalities and limitations of tracking and gating techniques in IMPT have also been reported by the group at Paul Scherrer Institute $(41,42)$.

Another key aspect is plan robustness, meaning the susceptibility of the nominal treatment plan due to uncertainties (e.g., setup uncertainties) or motion. Some treatment planning systems already allow the evaluation of plan robustness. Furthermore, consideration of robustness can not only be included in the treatment plan evaluation step but also in the plan optimization (robust optimization) (43-46). Usually, this will result in decreased quality of 
the nominal treatment plan in combination with smaller deviations between nominal and actual dose distribution under the influence of setup uncertainties and intra- and interfractional motion.

For PSPT, range and setup uncertainties can be taken into account by widening the aperture to ensure the lateral coverage in the presence of setup and/or in-patient target shifts. Furthermore, density changes lateral to the beam path, e.g., due to intrafractional motion, are mitigated by "smearing" the compensator. Range uncertainties are accounted for by increasing the nominal range. This method was investigated by several groups $(9,47)$ and is widely accepted in PSPT in the clinic $(8,48-50)$.

For PBS, no physical devices are used. Consequently, a different method needs to be applied to compensate for range and setup uncertainties. Laterally, an increased treatment area adding a margin to the tumor volume is applicable. For range uncertainties, a more sophisticated approach is the use of field-specific PTVs that take into account the influences of range uncertainty for each treatment field separately $(10,51,52)$. In comparison to a uniform margin extension, this leads to a PTV that can compensate for the specific requirements in proton therapy and thus preserves the target coverage in the presence of uncertainties.

\section{(IN SILICO) COMPARATIVE STUDIES}

Various institutions employing particle therapy or in the process of setting up the facility have published findings on comparative in silico planning studies using different photon and proton delivery techniques. Kase et al. (53) compared PSPT with IMPT in a variety of primary tumor sites, including NSCLC. IMPT resulted in lower dose to organs at risk, specifically, the high dose to the skin, the D20 to the normal lung, and the spinal cord.

Early-stage NSCLC. Wang and colleagues (54) from the Proton Medical Research Center compared 3D-CRT to PSPT in 24 patients with peripheral stage I NSCLC. Two to four proton beam ports were used and irradiation was applied at end exhalation. Photons were delivered using 5 to 7 coplanar ports covering the same clinical and planning target volume (CTV and PTV, respectively). The prescribed dose was $66 \mathrm{GyE}$ in 10 fractions at the isocenter. While the $90 \%$ isodose line covered $>99 \%$ of the CTV for both treatment modalities, the $95 \%$ isodose line covered only $86.4 \%$ of the CTV for proton plans and $43.2 \%$ for 3D-CRT plans. Organ at risk (OAR) doses, specifically, lungs, heart, esophagus, and spinal cord, were significantly lower for the proton beam technique.

The Mayo Clinic Group (55) generated treatment plans for eight stage I NSCLC patients with peripheral lung nodules using photon SBRT, and one-, two-, and three-field passively scattered or actively scanned proton beams. For SBRT $(3 \times 20 \mathrm{~Gy}), 10$ or more non-coplanar beams were manually selected to achieve optimal PTV coverage while minimizing dose to the OARs. Plans were normalized to isocenter with the prescription isodose line covering 95\% or more of the PTV. For proton beam treatment, beam direction was manually optimized to maximize access to the tumor, while minimizing exposure to OARs and adjacent normal tissues. Proton beam plans demonstrated significantly lower maximum and higher minimum PTV doses compared with SBRT. With the exception of the three-field actively scanned approach, the maximum dose $2 \mathrm{~cm}$ from the PTV was significantly higher with proton beams. The doses to OARs (lungs, spinal cord, heart, bronchial tree, esophagus, skin, and ribs) were generally lower with protons than with photons. Using actively scanned beams, the maximum dose to the PTV, V30Gy, and the dose to any tissue $2 \mathrm{~cm}$ from the PTV decreased, while the minimum dose to the PTV increased.

Similarly, Kadoya et al. (56) studied 21 patients with peripheral stage I NSCLC, delivering a dose of $66 \mathrm{GyE}$ in 10 fractions during maximal expiration using SBRT (7-8 non-coplanar 4-MV photon beams) or PSPT (2-3 directions). While the dose to the PTV was non-significantly different, the dose to the lung was significantly lower with the PSPT technique. The authors concluded that PSPT may be advantageous for large or multiple PTVs.

For stage I NSCLC, PBT may provide the greatest dosimetric and clinical benefit for patients with centrally located tumors given the higher reported toxicity when delivering SBRT for tumors in this region. For centrally and superiorly located stage I NSCLC, Register et al. (57) compared SBRT with PSPT and IMPT. SBRT was prescribed to $50 \mathrm{~Gy}$ in 12.5-Gy fractions, normalized such that $95 \%$ of the PTV received $100 \%$ of the prescribed dose. Each PSPT plan was created with three to four coplanar beam angles in an attempt to minimize the exit dose into the lung parenchyma. The same beam angels were used for generating the IMPT plans. Only 6 of 15 photon SBRT plans satisfied PTV coverage and all normal tissue dose constraints, compared to 12 PSPT and 14 IMPT plans.

A significant reduction in the normal tissue dose by passively scattered protons (66 GyE) compared to 3D-CRT or IMRT photon treatment ( $66 \mathrm{~Gy}$ ) was reported by Chang et al. (58) for stage I and stage IIIA/B NSCLC patients. For early-stage NSCLC $(n=10)$, the DVH comparison revealed a reduction in dose to the ipsilateral and contralateral lungs, heart, spinal cord, and esophagus with protons. For locally advanced patients $(n=15)$ in all cases, the doses to lung, heart, esophagus, spinal cord, and integral dose were lower with proton therapy than both 3D-CRT and IMRT photon modalities. This dosimetric benefits of protons over photons persisted even when escalating the PBT doses to 87.5 GyE for stage I NSCLC and 74 GyE for stage III NSCLC compared with 66 Gy for all photon plans.

An in silico planning study comparing modern photon techniques with protons and carbon ions in 25 stage I NSCLC patients is currently being conducted by the ROCOCO consortium (NCT02038413).

Locally advanced NSCLC. Proton beam therapy may be advantageous for locally advanced NSCLC patients delivering either the same dose with less toxicity or a higher dose under isotoxic conditions. Representative images showing plan comparisons for patients with locally advanced NSCLC are shown in Figures 1 and 2. In the first, PSPT spares significant volumes of heart, esophagus, and lung relative to IMRT. In the latter comparing IMRT, PSPT, and PBS using SFUD, the dose to the heart, spinal cord, and contralateral lung is significantly reduced using (advanced) proton techniques.

The MD Anderson Cancer Center conducted a prospective phase II clinical study combining chemotherapy with PSPT in 44 unresectable NSCLC patients (details and outcome of the study 

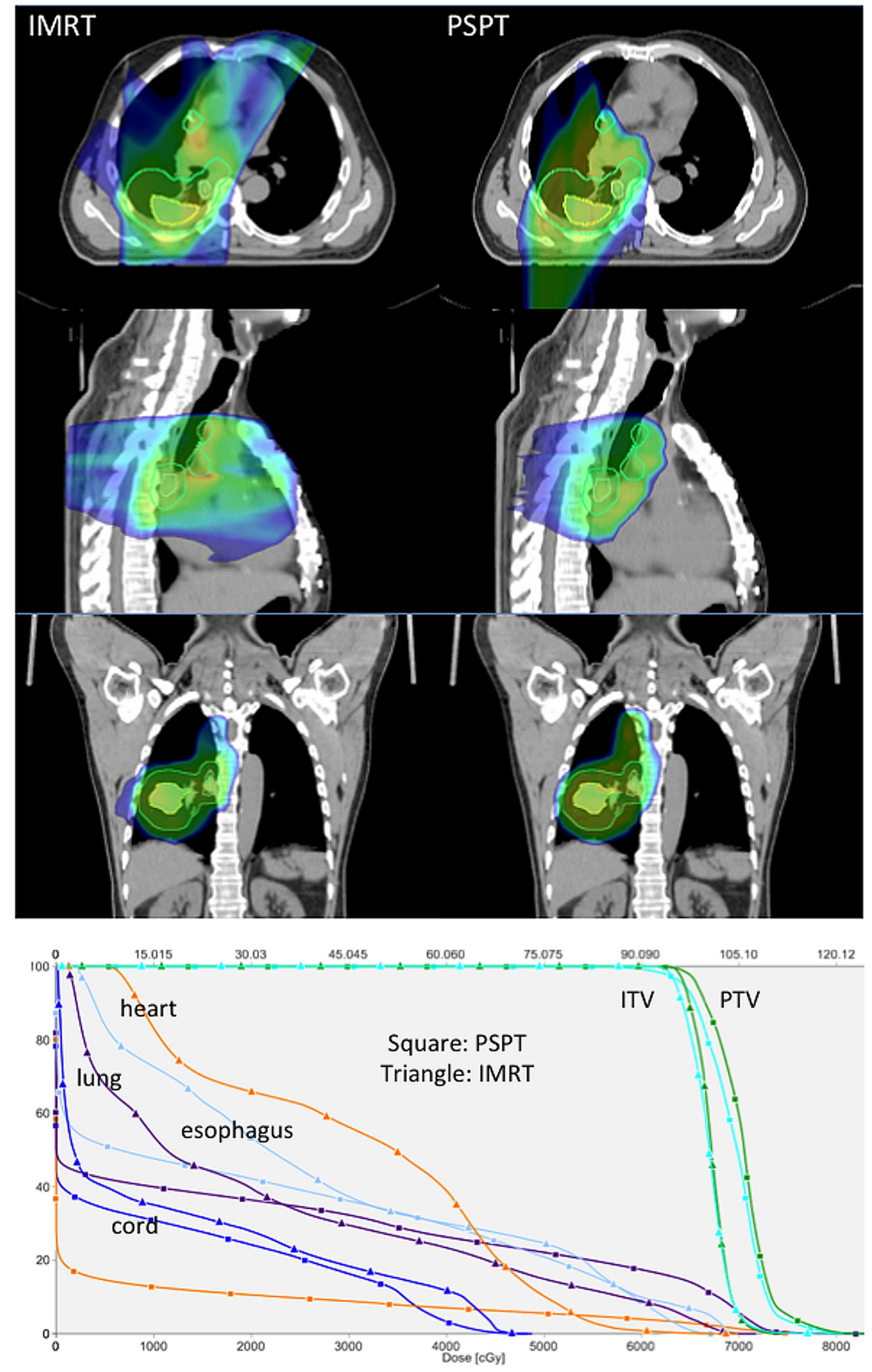

FIGURE 1 | Treatment planning images and dose-volume histogram comparison (Hospital of the University of Pennsylvania). Representative treatment planning images for a patient with locally advanced non-small cell lung cancer with a right lower lobe primary tumor (iGTV primary tumor depicted as a yellow contour) and multi-station mediastinal nodal metastasis (Continued) 


\section{FIGURE 1 | Continued}

(iGTV nodal metastasis depicted as a yellow contour). The composite PTV is depicted as a cyan contour. Comparative plans for IMRT (left) and proton beam therapy (right) are depicted in the axial planes (top row), sagittal planes (middle row), and coronal planes (bottom row) delivering 66.6 Gy (IMRT) or 66.6 GyE (proton therapy) in 37 fractions. Dose color wash coding: blue $=50 \%$ to red $=$ global max above $100 \%$. Representative dose-volume histograms for the same patient are also depicted showing dose to target volumes of PTV (green) and ITV (cyan) and dose to normal structures of heart (orange), total lung minus GTV (purple), esophagus (light blue), and spinal cord (dark blue) for IMRT (triangle) and proton (square) plans.

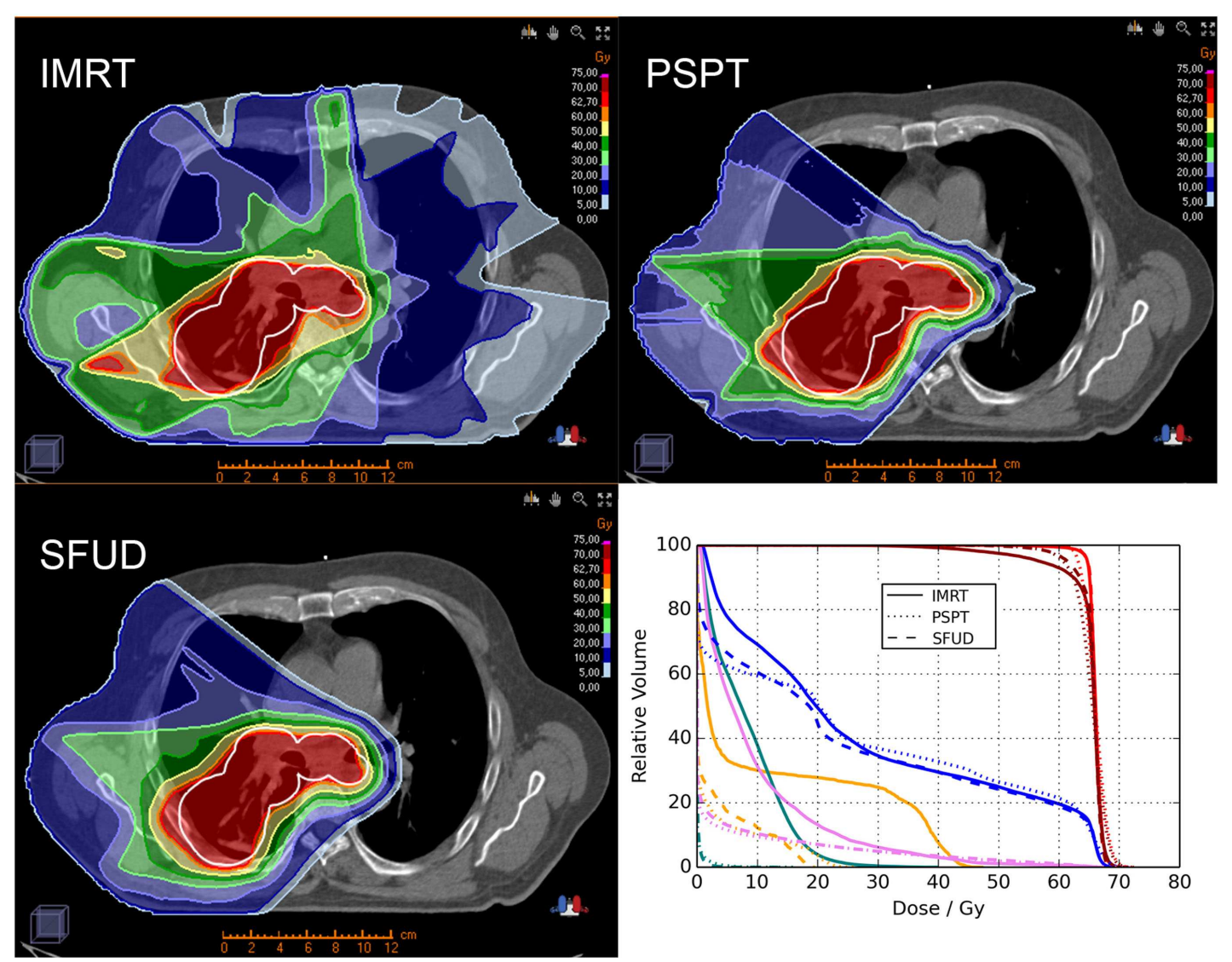

FIGURE 2 |Treatment plan comparison between IMRT (left upper row), PSPT (right upper row) and PBS using SFUD (left lower row) in an advanced-stage NSCLC patient (OncoRay, Dresden). The planning target volume (PTV) is represented by the white contour. Furthermore a dose-volume histogram (DVH; right lower row) analysis is illustrated for the three treatment techniques showing the clinical target volume (CTV; red), PTV (dark red), ipsilateral lung (blue), spinal cord (orange), heart (pink), and contralateral lung (turquois). described earlier (32). Koay et al. (33) published an analysis for the nine out of 44 patients for whom the plans were adapted during the treatment course. While the authors observed profound differences in the dose to the esophagus and spinal cord, no statistical comparison of the adapted versus non-adapted patients was performed. For two of the nine patients, the internal CTV coverage would have decreased significantly without adaptation. Remarkably, the reported toxicity was higher among the patients treated with adaptive plans, although statistical comparison was again lacking. Treatment outcome was not significantly different between the treatment strategies $(p>0.05)$.

Zhang et al. (59) compared IMPT with PSPT and IMRT in 20 NSCLC patients with extensive stage IIIB disease. Using IMRT, the selected patients had no or borderline tolerance to IMRT at $60-63$ Gy when keeping within the normal tissue dose constraints. IMPT succeeded in sparing more lung, heart, spinal cord, and esophagus when maintaining the prescribed dose. IMPT was even feasible when escalating the dose up to $83.5 \mathrm{~Gy}$ (mean maximum tolerated dose $74 \mathrm{~Gy}$ ), whereas PSPT was limited by the esophagus dose constraint (74 Gy). The University of Florida Proton Therapy Institute (60) compared 3D-CRT with IMRT and PSPT in eight NSCLC patients. The target dose prescription was achieved in all patients using either of the techniques. Compared to the photon techniques, PSPT considerably reduced the dose to radiation-sensitive normal structures: normal lung V20Gy by median 29\% for 3D-CRT and 26\% for IMRT, mean lung dose by 33 and $31 \%$, and V10Gy bone marrow by 30 and $27 \%$, respectively. This may offer an advantage in patients undergoing sequential or 
concurrent radiochemotherapy. In a multicentric in silico clinical trial, the ROCOCO consortium compared photon (3D-CRT and IMRT) with proton (PSPT) treatment for stage IA-IIIB NSCLC patients delivering $70 \mathrm{~Gy}$ in 35 fractions (8). The integral dose for the photon techniques was higher than for the proton techniques, whereas the mean lung dose was lower for protons. For 10 patients, dose escalation to the primary tumor up to $87 \mathrm{~Gy}$ was feasible for all three modalities; however, the mean lung and integral doses were higher for photons than for protons.

\section{CONCLUSION}

Proton beam delivery has evolved rapidly over the last decade, enabling highly conformal treatment delivery with the possibility of escalating the dose to the primary tumor while maintaining the dose to the normal tissues, or maintaining the dose to the target volume and reducing the dose to the organs at risk. Dosimetric advantages of protons over photons have been demonstrated across NSCLC stages of disease in reducing doses to critical organs at risk, including the lungs, heart, esophagus, and spinal cord. PBT has also been used clinically in an increasing number of prospective studies for both stage I NSCLC and locally advanced NSCLC with a generally more favorable toxicity profile than what has been reported with historical photon studies. Particle beam treatment may prove to provide its greatest clinical benefit compared with IMRT and photon treatment techniques for patients with pretreatment severe functional impairments (e.g., poor lung function, preexisting lung disease), when delivering escalating doses of radiation therapy or using radiation therapy as part of trimodality therapy combined with chemotherapy and surgery, and for definitive re-treatment of NSCLC patients with a local/locoregional recurrence following prior radiotherapy. From the in silico trials we have gathered, we can conclude that the dose distributions with IMPT are superior to those of the photon treatments and the passive scattering technique. Until now, PSPT has predominantly been used in the clinic instead of IMPT. It is likely that a more widespread introduction of IMPT can provide further improvement in lung cancer outcome and/or decreased toxicity, through increased precision and personalized treatment, provided that range uncertainties are well accounted for.

\section{REFERENCES}

1. DeSantis CE, Lin CC, Mariotto AB, Siegel RL, Stein KD, Kramer JL, et al. Cancer treatment and survivorship statistics, 2014. CA Cancer J Clin (2014) 64(4):252-71. doi:10.3322/caac. 21235

2. Auperin A, Le Pechoux C, Rolland E, Curran WJ, Furuse K, Fournel P, et al. Meta-analysis of concomitant versus sequential radiochemotherapy in locally advanced non-small-cell lung cancer. J Clin Oncol (2010) 28(13):2181-90. doi:10.1200/JCO.2009.26.2543

3. Machtay M, Paulus R, Moughan J, Komaki R, Bradley JE, Choy H, et al. Defining local-regional control and its importance in locally advanced non-small cell lung carcinoma. J Thorac Oncol (2012) 7(4):716-22. doi:10.1097/JTO. 0b013e3182429682

4. Kong FM, Zhao J, Wang J, Faivre-Finn C. Radiation dose effect in locally advanced non-small cell lung cancer. J Thorac Dis (2014) 6(4):336-47. doi:10. 3978/j.issn.2072-1439.2014.01.23

5. van Baardwijk A, Wanders S, Boersma L, Borger J, Ollers M, Dingemans AM, et al. Mature results of an individualized radiation dose prescription study based on normal tissue constraints in stages I to III non-small-cell lung cancer. J Clin Oncol (2010) 28(8):1380-6. doi:10.1200/JCO.2009.24.7221
6. Baumann M, Herrmann T, Koch R, Matthiessen W, Appold S, Wahlers B, et al. Final results of the randomized phase III CHARTWEL-trial (ARO 971) comparing hyperfractionated-accelerated versus conventionally fractionated radiotherapy in non-small cell lung cancer (NSCLC). Radiother Oncol (2011) 100(1):76-85. doi:10.1016/j.radonc.2011.06.031

7. Hoffmann AL, Troost EG, Huizenga H, Kaanders JH, Bussink J. Individualized dose prescription for hypofractionation in advanced non-small-cell lung cancer radiotherapy: an in silico trial. Int J Radiat Oncol Biol Phys (2012) 83(5):1596-602. doi:10.1016/j.ijrobp.2011.10.032

8. Roelofs E, Engelsman M, Rasch C, Persoon L, Qamhiyeh S, de Ruysscher D, et al. Results of a multicentric in silico clinical trial (ROCOCO): comparing radiotherapy with photons and protons for non-small cell lung cancer. J Thorac Oncol (2012) 7(1):165-76. doi:10.1097/JTO.0b013e31823529fc

9. Moyers MF, Miller DW, Bush DA, Slater JD. Methodologies and tools for proton beam design for lung tumors. Int J Radiat Oncol Biol Phys (2001) 49(5):1429-38. doi:10.1016/S0360-3016(00)01555-8

10. Park PC, Zhu XR, Lee AK, Sahoo N, Melancon AD, Zhang L, et al. A beamspecific planning target volume (PTV) design for proton therapy to account for setup and range uncertainties. Int J Radiat Oncol Biol Phys (2012) 82(2):e329-36. doi:10.1016/j.ijrobp.2011.05.011

11. van Baardwijk A, Tome WA, van Elmpt W, Bentzen SM, Reymen B, Wanders R, et al. Is high-dose stereotactic body radiotherapy (SBRT) for stage I non-small cell lung cancer (NSCLC) overkill? A systematic review. Radiother Oncol (2012) 105(2):145-9. doi:10.1016/j.radonc.2012.09.008

12. Palma D, Visser O, Lagerwaard FJ, Belderbos J, Slotman B, Senan S. Treatment of stage I NSCLC in elderly patients: a population-based matched-pair comparison of stereotactic radiotherapy versus surgery. Radiother Oncol (2011) 101(2):240-4. doi:10.1016/j.radonc.2011.06.029

13. Palma D, Senan S. Stereotactic radiation therapy: changing treatment paradigms for stage I nonsmall cell lung cancer. Curr Opin Oncol (2011) 23(2):133-9. doi:10.1097/CCO.0b013e328341ee11

14. Bush DA, Slater JD, Shin BB, Cheek G, Miller DW, Slater JM. Hypofractionated proton beam radiotherapy for stage I lung cancer. Chest (2004) 126(4):1198-203. doi:10.1378/chest.126.4.1198

15. Nihei K, Ogino T, Ishikura S, Nishimura H. High-dose proton beam therapy for stage I non-small-cell lung cancer. Int J Radiat Oncol Biol Phys (2006) 65(1):107-11. doi:10.1016/j.ijrobp.2005.10.031

16. Hata M, Tokuuye K, Kagei K, Sugahara S, Nakayama H, Fukumitsu N, et al. Hypofractionated high-dose proton beam therapy for stage I non-small-cell lung cancer: preliminary results of a phase I/II clinical study. Int J Radiat Oncol Biol Phys (2007) 68(3):786-93. doi:10.1016/j.ijrobp.2006.12.063

17. Nakayama H, Sugahara S, Tokita M, Satoh H, Tsuboi K, Ishikawa S, et al. Proton beam therapy for patients with medically inoperable stage I non-small-cell lung cancer at the University of Tsukuba. Int J Radiat Oncol Biol Phys (2010) 78(2):467-71. doi:10.1016/j.ijrobp.2009.07.1707

18. Bush DA, Cheek G, Zaheer S, Wallen J, Mirshahidi H, Katerelos A, et al. Highdose hypofractionated proton beam radiation therapy is safe and effective for central and peripheral early-stage non-small cell lung cancer: results of a 12year experience at Loma Linda University Medical Center. Int J Radiat Oncol Biol Phys (2013) 86(5):964-8. doi:10.1016/j.ijrobp.2013.05.002

19. Kanemoto A, Okumura T, Ishikawa H, Mizumoto M, Oshiro Y, Kurishima K, et al. Outcomes and prognostic factors for recurrence after high-dose proton beam therapy for centrally and peripherally located stage I non - small-cell lung cancer. Clin Lung Cancer (2014) 15(2):e7-12. doi:10.1016/j.cllc.2013.11.002

20. Miyamoto T, Yamamoto N, Nishimura H, Koto M, Tsujii H, Mizoe JE, et al. Carbon ion radiotherapy for stage I non-small cell lung cancer. Radiother Oncol (2003) 66(2):127-40. doi:10.1016/S0167-8140(02)00367-5

21. Miyamoto T, Baba M, Sugane T, Nakajima M, Yashiro T, Kagei K, et al. Carbon ion radiotherapy for stage I non-small cell lung cancer using a regimen of four fractions during 1 week. J Thorac Oncol (2007) 2(10):916-26. doi:10.1097/JTO.0b013e3181560a68

22. Miyamoto T, Baba M, Yamamoto N, Koto M, Sugawara T, Yashiro T, et al. Curative treatment of Stage I non-small-cell lung cancer with carbon ion beams using a hypofractionated regimen. Int J Radiat Oncol Biol Phys (2007) 67(3):750-8. doi:10.1016/j.ijrobp.2006.10.006

23. Sugane T, Baba M, Imai R, Nakajima M, Yamamoto N, Miyamoto T, et al. Carbon ion radiotherapy for elderly patients 80 years and older with stage I non-small cell lung cancer. Lung Cancer (2009) 64(1):45-50. doi:10.1016/j.lungcan.2008. 07.007 
24. Iwata H, Murakami M, Demizu Y, Miyawaki D, Terashima K, Niwa Y, et al. High-dose proton therapy and carbon-ion therapy for stage I nonsmall cell lung cancer. Cancer (2010) 116(10):2476-85. doi:10.1002/cncr.24998

25. Iwata H, Demizu Y, Fujii O, Terashima K, Mima M, Niwa Y, et al. Longterm outcome of proton therapy and carbon-ion therapy for large (T2aT2bN0M0) non-small-cell lung cancer. J Thorac Oncol (2013) 8(6):726-35. doi:10.1097/JTO.0b013e318288ab02

26. Bush DA, Slater JD, Bonnet R, Cheek GA, Dunbar RD, Moyers M, et al. Protonbeam radiotherapy for early-stage lung cancer. Chest (1999) 116(5):1313-9. doi:10.1378/chest.116.5.1313

27. Shioyama Y, Tokuuye K, Okumura T, Kagei K, Sugahara S, Ohara K, et al. Clinical evaluation of proton radiotherapy for non-small-cell lung cancer. Int J Radiat Oncol Biol Phys (2003) 56(1):7-13. doi:10.1016/S0360-3016(02)04416-4

28. Timmerman R, Paulus R, Galvin J, Michalski J, Straube W, Bradley J, et al. Stereotactic body radiation therapy for inoperable early stage lung cancer. JAMA (2010) 303(11):1070-6. doi:10.1001/jama.2010.261

29. Timmerman R, McGarry R, Yiannoutsos C, Papiez L, Tudor K, DeLuca J, et al. Excessive toxicity when treating central tumors in a phase II study of stereotactic body radiation therapy for medically inoperable early-stage lung cancer. J Clin Oncol (2006) 24(30):4833-9. doi:10.1200/JCO.2006.07.5937

30. Oshiro Y, Mizumoto M, Okumura T, Hashimoto T, Fukumitsu N, Ohkawa A, et al. Results of proton beam therapy without concurrent chemotherapy for patients with unresectable stage III non-small cell lung cancer. J Thorac Oncol (2012) 7(2):370-5. doi:10.1097/JTO.0b013e31823c485f

31. Nakayama H, Satoh H, Sugahara S, Kurishima K, Tsuboi K, Sakurai H, et al. Proton beam therapy of stage II and III non-small-cell lung cancer. Int J Radiat Oncol Biol Phys (2011) 81(4):979-84. doi:10.1016/j.ijrobp.2010.06.024

32. Chang JY, Komaki R, Lu C, Wen HY, Allen PK, Tsao A, et al. Phase 2 study of high-dose proton therapy with concurrent chemotherapy for unresectable stage III nonsmall cell lung cancer. Cancer (2011) 117(20):4707-13. doi: $10.1002 / \mathrm{cncr} .26080$

33. Koay EJ, Lege D, Mohan R, Komaki R, Cox JD, Chang JY. Adaptive/nonadaptive proton radiation planning and outcomes in a phase II trial for locally advanced non-small cell lung cancer. Int J Radiat Oncol Biol Phys (2012) 84(5):1093-100. doi:10.1016/j.ijrobp.2012.02.041

34. Xiang ZL, Erasmus J, Komaki R, Cox JD, Chang JY. FDG uptake correlates with recurrence and survival after treatment of unresectable stage III non-small cell lung cancer with high-dose proton therapy and chemotherapy. Radiat Oncol (2012) 7:144. doi:10.1186/1748-717X-7-144

35. Matney J, Park PC, Bluett J, Chen YP, Liu W, Court LE, et al. Effects of respiratory motion on passively scattered proton therapy versus intensity modulated photon therapy for stage III lung cancer: are proton plans more sensitive to breathing motion? Int J Radiat Oncol Biol Phys (2013) 87(3):576-82. doi:10.1016/j.ijrobp.2013.07.007

36. Bert C, Grozinger SO, Rietzel E. Quantification of interplay effects of scanned particle beams and moving targets. Phys Med Biol (2008) 53(9):2253-65. doi:10.1088/0031-9155/53/9/003

37. Li Y, Kardar L, Li X, Li H, Cao W, Chang JY, et al. On the interplay effects with proton scanning beams in stage III lung cancer. Med Phys (2014) 41(2):021721. doi:10.1118/1.4862076

38. Grassberger C, Dowdell S, Lomax A, Sharp G, Shackleford J, Choi N, et al. Motion interplay as a function of patient parameters and spot size in spot scanning proton therapy for lung cancer. Int J Radiat Oncol Biol Phys (2013) 86(2):380-6. doi:10.1016/j.ijrobp.2013.01.024

39. Dowdell S, Grassberger C, Sharp GC, Paganetti H. Interplay effects in proton scanning for lung: a 4D Monte Carlo study assessing the impact of tumor and beam delivery parameters. Phys Med Biol (2013) 58(12):4137-56. doi:10.1088/0031-9155/58/12/4137

40. Munck af Rosenschold P, Aznar MC, Nygaard DE, Persson GF, Korreman SS, Engelholm SA, et al. A treatment planning study of the potential of geometrical tracking for intensity modulated proton therapy of lung cancer. Acta Oncol (2010) 49(7):1141-8. doi:10.3109/0284186X.2010.500620

41. van de Water S, Kreuger R, Zenklusen S, Hug E, Lomax AJ. Tumour tracking with scanned proton beams: assessing the accuracy and practicalities. Phys Med Biol (2009) 54(21):6549-63. doi:10.1088/0031-9155/54/21/007

42. Schatti A, Zakova M, Meer D, Lomax AJ. The effectiveness of combined gating and re-scanning for treating mobile targets with proton spot scanning.
An experimental and simulation-based investigation. Phys Med Biol (2014) 59(14):3813-28. doi:10.1088/0031-9155/59/14/3813

43. Liu W, Frank SJ, Li X, Li Y, Park PC, Dong L, et al. Effectiveness of robust optimization in intensity-modulated proton therapy planning for head and neck cancers. Med Phys (2013) 40(5):051711. doi:10.1118/1.4801899

44. Engelsman M, Schwarz M, Dong L. Physics controversies in proton therapy. Semin Radiat Oncol (2013) 23(2):88-96. doi:10.1016/j.semradonc. 2012.11.003

45. Stuschke M, Kaiser A, Pottgen C, Lubcke W, Farr J. Potentials of robust intensity modulated scanning proton plans for locally advanced lung cancer in comparison to intensity modulated photon plans. Radiother Oncol (2012) 104(1):45-51. doi:10.1016/j.radonc.2012.03.017

46. Unkelbach J, Bortfeld T, Martin BC, Soukup M. Reducing the sensitivity of IMPT treatment plans to setup errors and range uncertainties via probabilistic treatment planning. Med Phys (2009) 36(1):149-63. doi:10.1118/ 1.3021139

47. Engelsman M, Rietzel E, Kooy HM. Four-dimensional proton treatment planning for lung tumors. Int J Radiat Oncol Biol Phys (2006) 64(5):1589-95. doi:10.1016/j.ijrobp.2005.12.026

48. Hoppe BS, Flampouri S, Henderson RH, Pham D, Bajwa AA, D’Agostino H, et al. Proton therapy with concurrent chemotherapy for non-small-cell lung cancer: technique and early results. Clin Lung Cancer (2012) 13(5):352-8. doi:10.1016/j.cllc.2011.11.008

49. Seco J, Panahandeh HR, Westover K, Adams J, Willers H. Treatment of nonsmall cell lung cancer patients with proton beam-based stereotactic body radiotherapy: dosimetric comparison with photon plans highlights importance of range uncertainty. Int J Radiat Oncol Biol Phys (2012) 83(1):354-61. doi:10.1016/j.ijrobp.2011.05.062

50. Hui Z, Zhang X, Starkschall G, Li Y, Mohan R, Komaki R, et al. Effects of interfractional motion and anatomic changes on proton therapy dose distribution in lung cancer. Int J Radiat Oncol Biol Phys (2008) 72(5):1385-95. doi:10.1016/j.ijrobp.2008.03.007

51. Knopf AC, Boye D, Lomax A, Mori S. Adequate margin definition for scanned particle therapy in the incidence of intrafractional motion. Phys Med Biol (2013) 58(17):6079-94. doi:10.1088/0031-9155/58/17/6079

52. Bert C, Rietzel E. 4D treatment planning for scanned ion beams. Radiat Oncol (2007) 2:24. doi:10.1186/1748-717X-2-24

53. Kase Y, Yamashita H, Fuji H, Yamamoto Y, Pu Y, Tsukishima C, et al. A treatment planning comparison of passive-scattering and intensity-modulated proton therapy for typical tumor sites. J Radiat Res (2012) 53(2):272-80. doi:10.1269/jrr.11136

54. Wang C, Nakayama H, Sugahara S, Sakae T, Tokuuye K. Comparisons of dosevolume histograms for proton-beam versus 3-D conformal $\mathrm{x}$-ray therapy in patients with stage I non-small cell lung cancer. Strahlenther Onkol (2009) 185(4):231-4. doi:10.1007/s00066-009-1923-X

55. Macdonald OK, Kruse JJ, Miller JM, Garces YI, Brown PD, Miller RC, et al. Proton beam radiotherapy versus three-dimensional conformal stereotactic body radiotherapy in primary peripheral, early-stage non-small-cell lung carcinoma: a comparative dosimetric analysis. Int J Radiat Oncol Biol Phys (2009) 75(3):950-8. doi:10.1016/j.ijrobp.2009.04.023

56. Kadoya N, Obata Y, Kato T, Kagiya M, Nakamura T, Tomoda T, et al. Dosevolume comparison of proton radiotherapy and stereotactic body radiotherapy for non-small-cell lung cancer. Int J Radiat Oncol Biol Phys (2011) 79(4):1225-31. doi:10.1016/j.ijrobp.2010.05.016

57. Register SP, Zhang X, Mohan R, Chang JY. Proton stereotactic body radiation therapy for clinically challenging cases of centrally and superiorly located stage I non-small-cell lung cancer. Int J Radiat Oncol Biol Phys (2011) 80(4):1015-22. doi:10.1016/j.ijrobp.2010.03.012

58. Chang JY, Zhang X, Wang X, Kang Y, Riley B, Bilton S, et al. Significant reduction of normal tissue dose by proton radiotherapy compared with three-dimensional conformal or intensity-modulated radiation therapy in stage I or stage III non-small-cell lung cancer. Int J Radiat Oncol Biol Phys (2006) 65(4):1087-96. doi:10.1016/j.ijrobp.2006.01.052

59. Zhang X, Li Y, Pan X, Xiaoqiang L, Mohan R, Komaki R, et al. Intensitymodulated proton therapy reduces the dose to normal tissue compared with intensity-modulated radiation therapy or passive scattering proton therapy and enables individualized radical radiotherapy for extensive stage IIIB 
non-small-cell lung cancer: a virtual clinical study. Int J Radiat Oncol Biol Phys (2010) 77(2):357-66. doi:10.1016/j.ijrobp.2009.04.028

60. Nichols RC, Huh SN, Henderson RH, Mendenhall NP, Flampouri S, Li Z, et al. Proton radiation therapy offers reduced normal lung and bone marrow exposure for patients receiving dose-escalated radiation therapy for unresectable stage iii non-small-cell lung cancer: a dosimetric study. Clin Lung Cancer (2011) 12(4):252-7. doi:10.1016/j.cllc.2011.03.027

Conflict of Interest Statement: The authors declare that the research was conducted in the absence of any commercial or financial relationships that could be construed as a potential conflict of interest.

Received: 31 July 2014; accepted: 07 October 2014; published online: 29 October 2014.
Citation: Wink KCJ, Roelofs E, Solberg T, Lin L, Simone CB II, Jakobi A, Richter C, Lambin P and Troost EGC (2014) Particle therapy for non-small cell lung tumors: where do we stand? A systematic review of the literature. Front. Oncol. 4:292. doi: 10.3389/fonc.2014.00292

This article was submitted to Radiation Oncology, a section of the journal Frontiers in Oncology.

Copyright $\odot 2014$ Wink, Roelofs, Solberg, Lin, Simone, Jakobi, Richter, Lambin and Troost. This is an open-access article distributed under the terms of the Creative Commons Attribution License (CC BY). The use, distribution or reproduction in other forums is permitted, provided the original author(s) or licensor are credited and that the original publication in this journal is cited, in accordance with accepted academic practice. No use, distribution or reproduction is permitted which does not comply with these terms. 